\title{
Development of Cotton Flower Picking Machine based on Machine Vision Technique
}

\author{
Alakh Patel \\ Department of ECE \\ Babaria Institute of Technology, Varnama
}

\author{
Jaymin Bhalani \\ Professor, Department of ECE \\ Babaria Institute of Technology, Varnama
}

\begin{abstract}
India is second largest country in production of cotton. But cotton production face problem of harvesting which is currently done by skilled human labour. Now actual problem is that no one wants to work in farm so one should always think about alternative way of harvesting cotton. One approach to solve this problem is by taking help from intelligent machine. Proposed work focused on introduction of image processing into cotton harvesting. Following proposed work contain one system which is combination of motion control and machine vision system by which cotton harvesting is made possible. Machine vision system takes real time image of cotton plant and processes it to find out cotton ball in it followed by mechanical arm which pick cotton from plant. System consider all the parameter like lighting ,background, leaves and other surrounding objects to the cotton plants. System equip with various sensor for movement along the farm. Attempt is to make one miniature model which work exactly like proposed system. This system can also use to harvest different fruits by making little change in image processing algorithm.
\end{abstract}

\section{Keywords}

Cotton Recognition, image segmentation, cotton harvesting robot, Cotton Picking Machine, Smart Harvester.

\section{INTRODUCTION}

Technology has large impact on agriculture productivity. Whether it is mechanical or compute technology, both have large application in agriculture. It can be used from plantation of crop to harvesting of crop. Harvesting is challenging procedure in today condition because it requires skilled labour, more time to harvest and also maintaining quality of product is important. So one can apply technology in harvesting, say for example now days there are so many equipment that are available for harvesting like cotton harvester, sugarcane harvester as shown in figure 1. But these types of machine are not intelligent or it require human interface for their operation.

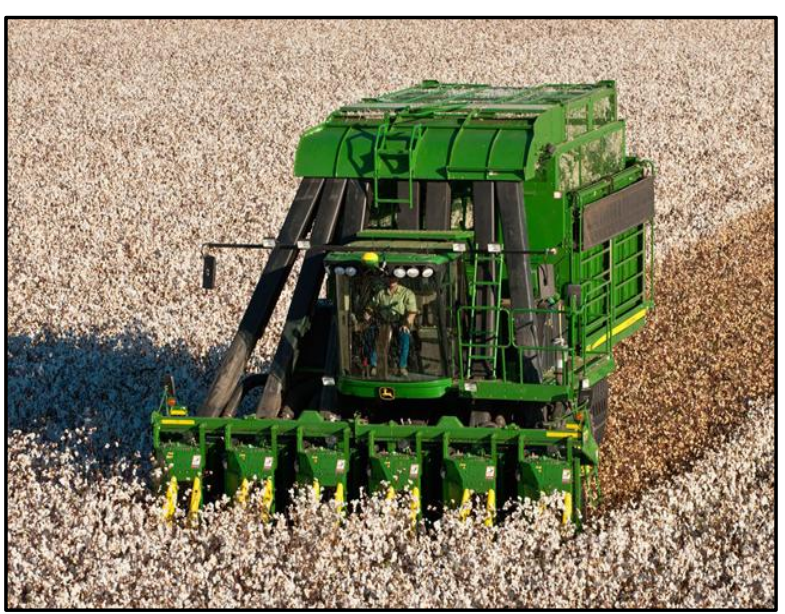

Figure 1: Cotton Harvester

In today world, machine vision or machine intelligence concept used in many area where machine works without human interface. Machine vision is the technology and methods used to provide imaging based automatic inspection and analysis for such application as automatic inspection, process control, and robot guidance, usually in industry. It use image based automatic inspection method. It can also consider as virtual vision in term of images \& data provided by sensor network. In simple language whatever human can see, machine can also see. Hence, it finds application in agriculture harvesting where machine have to take decision for harvesting.

Mulan Wang [1] proposed a research for intelligent cotton picking robot based on machine vision. In this, on the basis of the statistical analysis, a visual model for recognition of mature cotton was established and further optimized method increase the speed of operation. Yong Wang [2] proposed machine vision based cotton recognition for cotton harvesting robot. This method is based on color subtraction information of different parts of cotton. In order to increase accuracy rate of cotton recognition, dynamic freeman chain coding is used to remove noise. Yong Wang [3] proposed object recognition on cotton harvesting robot using human visual system. In this an algorithm based on the characteristic of HVS is proposed. In algorithm, the image was partitioned into many blocks of equal size. Mahua Bhattacharya [5] proposed Expert system design for cotton harvesting using shape and fractal features. They proposed image processing and soft computing based tools for analysis of the images of cotton bolls which are exactly in the mature form to be picked up. Yanan Li [6] proposed in field cotton detection via region based semantic image segmentation. This paper reports on a novel in field cotton detection via region based semantic image segmentation with two perspective of observation, including unsupervised region generation and supervised semantic labelling prediction. 
In proposed work "Cotton Flower Picking Machine Based on Machine Vision Technique" is present. As name suggest it harvest cotton flower, one at a time, using machine vision technique and robotic arm. System consist of camera to take image of cotton, robotic arm to pick cotton from plant, image processing algorithm to find cotton flower in image, motion control system to move along farm. Combining all system makes cotton flower picking machine. Although this machine is self-operated, it require small human interface for its operation. Human interface by means of taking machine to farm in which harvesting is perform and guide it along farm.

\section{COTTON FIELD ENVIRONMENT}

For analysis and observation of cotton plant and field, one cotton farm is chosen at Kasampur village of Karjan town in Vadodara district, Gujarat, India. Major Points of notice are how cotton plant is spaced among each other, how dense plant is and what is height and width of cotton plant etc. Although size and density of plant varied using different variety of seeds, proposed work is perform on chosen farm where plant is show in figure 2 .

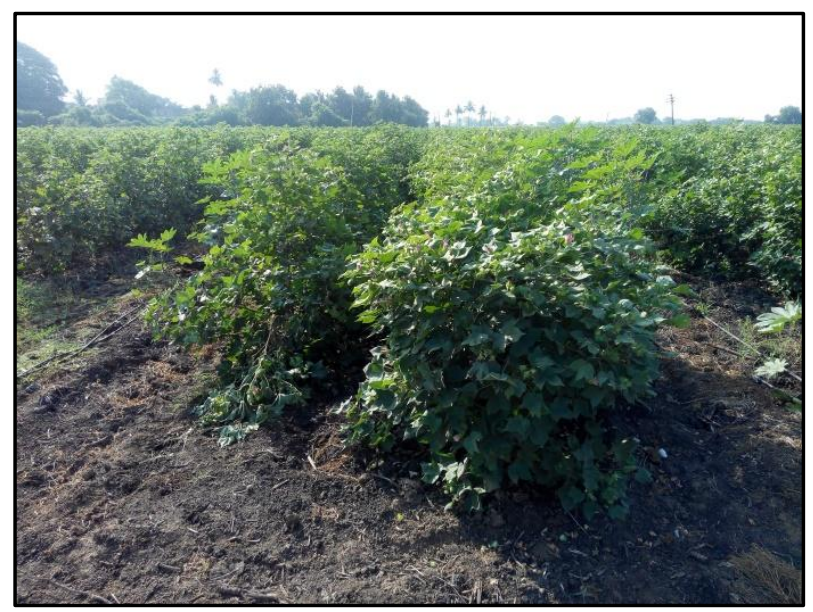

Figure 1 Cotton Plants at Experiment site

For image processing operation, about 300 image of cotton flower was captured during different sunlight condition. For example, some images captured at high sun light during 12 noon to $3 \mathrm{PM}$ while other are captured in morning and evening when sunlight is low.

\section{COTTON FLOWER PICKING MACHINE (CFPM)}

Cotton is one of the most important fiber and cash crop of India and plays a dominant role in the industrial and agricultural economy of the country. As mention earlier in introduction, traditional cotton harvesting can be replace using cotton harvesting robots based on machine vision. In this paper proposed method is termed as "Cotton Flower Picking Machine (CFPM).

\subsection{Block Diagram of CFPM}

CFPM is simply combination of machine vision system and motion control system with robotic arm as the end-effector. It uses MATLAB software as machine vision system along with use of external camera. Robotic arm is mechanical arm which can be used to pick detected cotton by means of cotton harvesting. Figure 3 shows block diagram of CFPM.

Camera is main component in any machine vision system using which images or video stream of object of interest in natural scene is capture. Usually it is CCD camera.

Machine vision is the technology and method used to provide imaging based automatic inspection and analysis for such application as automatic inspection, process control, and robot guidance usually in industry. Machine vision is closely related to image processing. In proposed work, MATLAB software is used which is propriety software i.e. one should have license to use this software form their organization.

Motion control system is nothing but simple 4 wheel robot that moves along farm. It consists of platform on which it carries controller and other subsystem like machine vision system, robotic arm, end effector and also power supply unit. In other words, motion control system provides forward and backward motion to whole cotton flower picking system in and out of farm.

A robotic arm is a type of mechanical arm, usually programmable, with similar function to a human arm, the arm may be the sum total of the mechanism or may be part of a more complex robot. In proposed work, robotic arm is of 3 DOF i.e. 3 degree of freedom with additional gripper. Dc servo motor is used for providing degree freedom to arm which is $9.4 \mathrm{~kg} . \mathrm{cm}$ torque servo motor with metal gear. It is just miniature model of robotic arm which shows how actual arm work and look like.

The Arduino Mega 2560 is a microcontroller board based on the ATmega2560. It has 54 digital input/output pins (of which 15 can be used as PWM outputs), 16 analog inputs, 4 UARTs (hardware serial ports), a $16 \mathrm{MHz}$ crystal oscillator, a USB connection, a power jack, and ICSP header and reset button.

Arduino is used to drive servo motor of robotic arm as well as to control movement of motion control system.

\subsection{Flow Chart of CFPM}

Figure 4 shows flowchart for proposed system "cotton flower picking machine". It contains various steps from taking images of cotton form field to harvest cotton by robotic arm.

First step in process is to take image of cotton plant in real time by using camera mounted on system. The camera is calibrated to take image of plant from specific distance and at specific angle. Every time camera take different position to capture image of cotton flower and thereby covering entire plant using predetermined path. 


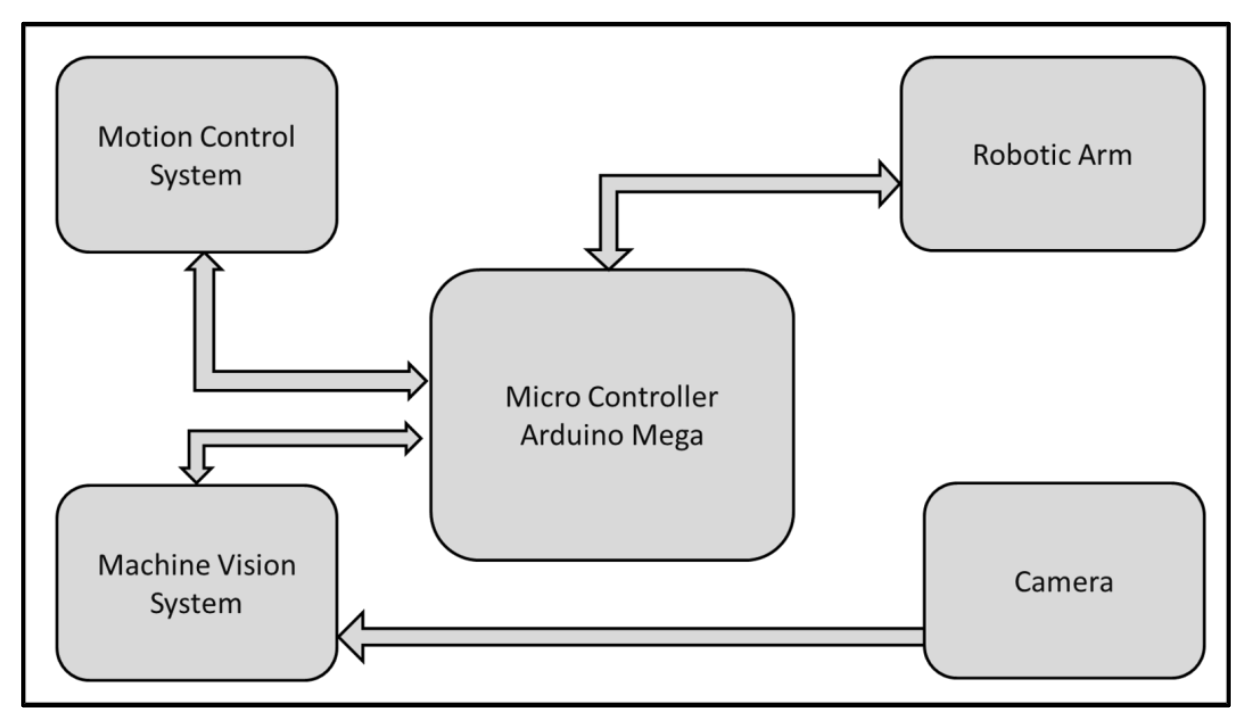

Figure 2 Block Diagram of CFPM

After that, image is given to machine vision system which process image by the means of image processing to find cotton flower in it. Image segmentation includes operation like object detection and shape detection etc. The goal of image segmentation is to simplify or change the representation of image into something that is more meaningful and easier to analyse. Image segmentation is typically used to locate object and boundaries in image.

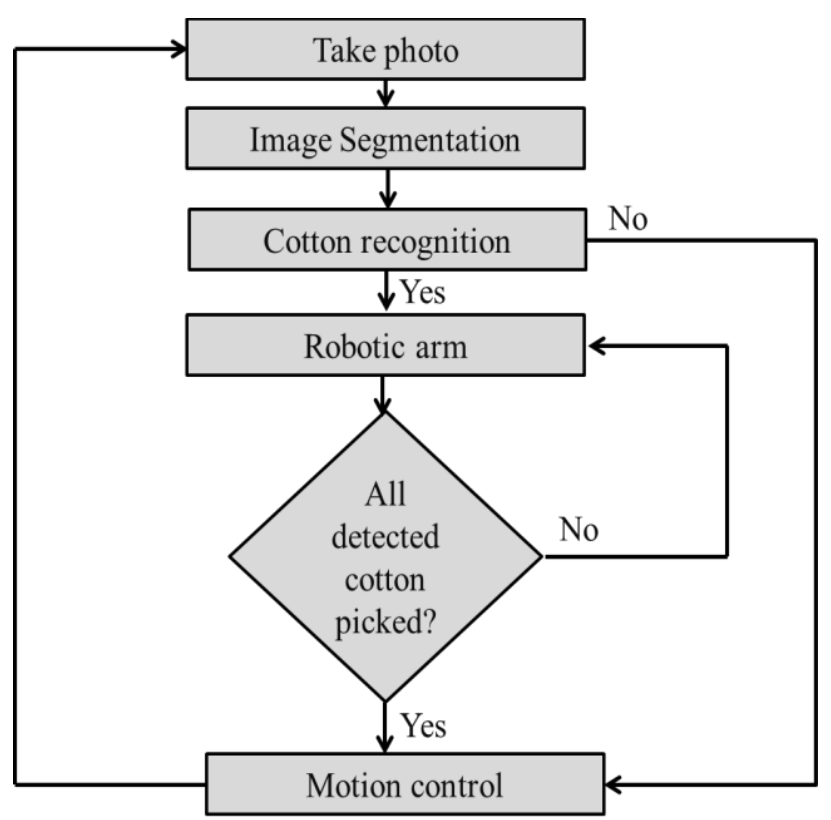

Figure 3 Flow Chart of CFPM

\subsection{Image Processing Technique}

In computer vision, image segmentation is the process of partitioning a digital image into multiple segments. The goal of segmentation is to simplify and change the representation of an image into something that is more meaningful and easier to analyse. Image segmentation is typically used to locate objects and boundaries in image. In proposed work, $\mathrm{k}-$ means
Clustering method of segmentation used to locate object in the cotton flower images captured by camera.

\subsubsection{K-Means Clustering Method of}

\section{Segmentation.}

$\mathrm{K}$-means clustering is a method of vector quantization, originally form signal processing, that is popular for cluster analysis in data mining. K-means clustering aims to partition $\mathrm{n}$ observations into $\mathrm{k}$ cluster in which each observation belongs to the cluster with the nearest mean, serving as prototype of the cluster. This results in a partitioning of the data space into voronoi cells.

Clustering is a way to separate groups of objects. K-means clustering treats each object as having a location in space. It finds partitions such that objects within each cluster are close to each other as possible, and as far from objects in other cluster as possible. K-means clustering requires that you specify the number of clusters to be partitioned and a distance metric to quantify how close two objects are to each other.

The algorithm iterates over following steps:

1. Initialization: $\mathrm{k}$ chosen, an initial set of $\mathrm{k}$ so-called centroid, i.e. virtual points in the data space are randomly created.

2. Compute the distance of each point from each cluster by computing its distance from the corresponding cluster mean. Assign each point to the cluster it is nearest to.

3. The position of the centroid is updated by means of the data points assigned to that cluster. In other words, the centroid is moved toward the centre of its assigned points.

4. Step 2 and 3 are performed until no centroid was shifted in one iteration. The algorithm is stopped when the minimum shift is below a threshold. 


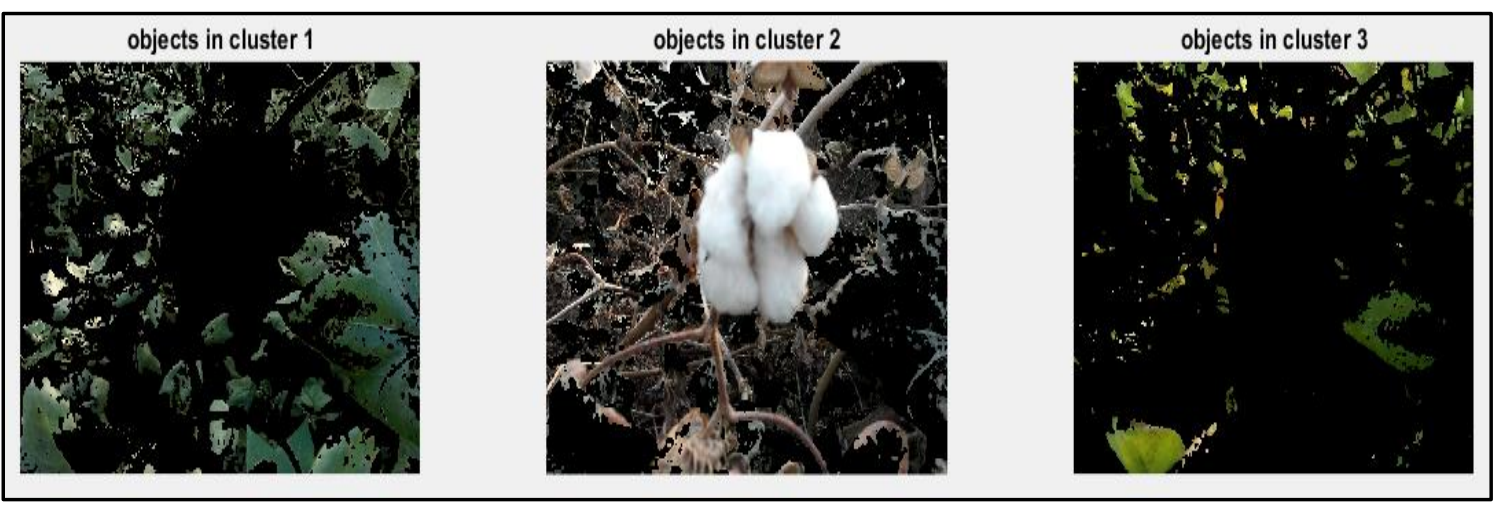

Figure 4 Detected Cotton using K-means Clustering Algorithm

\subsubsection{Gamma Correction for Cotton Detection}

Gamma correction is the name of a nonlinear operation used to encode and decode luminance value in video or still image system. Gamma correction is in simplest cases defined by following power-law expression.

$$
V_{\text {out }}=A V_{\text {in }}^{\gamma}
$$

Where the non-negative real input value $V_{i n}$ is raised to the power $\gamma$ and multiplied by the constant $\mathrm{A}$, to get the output value $V_{\text {out }}$. In the common case of $\mathrm{A}=1$, inputs and outputs are typically in the range $0-1$. A gamma value $y<1$ is sometimes called an encoding gamma and the process of encoding with this compressive power-law nonlinearity is called gamma compression, conversely a gamma value $y>1$ is called a decoding gamma and the application of the expansive power-law nonlinearity is called gamma expansion.

k-means clustering detect cotton flower form image with some noise surrounding cotton flower in terms of leaf and stem of plant. This noise can be eliminated by performing gamma correction on clustered image.

For performing gamma correction, clustered image, which RGB image, is first converted in grayscale image. Then gamma adjust is applied on grayscale image to remove noise and distinguished cotton flower from background. Figure 6 shows detected cotton flower image which gamma adjusts.
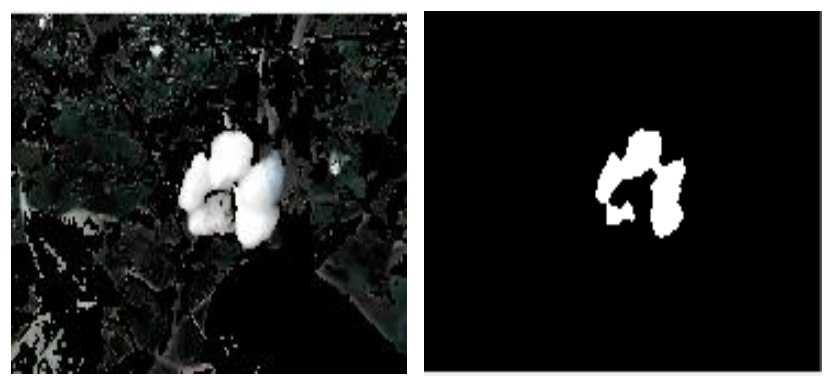

Figure 5 Clustered Image \& Gamma Corrected Image

\subsubsection{Result of $K-$ means clustering}

About 40 image of cotton flower are tested using k-means algorithm and result shows that it gives best accuracy with fast processing. Table 1 show summary of result k-means clustering.

Table 1 Result

\begin{tabular}{|c|c|c|c|}
\hline \multicolumn{4}{|c|}{ k-means Clustering } \\
\hline TP / True & TN / False & Noise & Accuracy \\
\hline 33 & 6 & 1 & $97 \%$ \\
\hline
\end{tabular}

Here TP- True Positive means correct detection when cotton flower is there.

TN - True Negative means correct detection when no cotton flower

Noise - other than cotton flower, background and other object also appear in image.

\section{PROPOSED MODEL FOR CFPM}

In following proposed work, attempt is to make miniature model of CFPM which gives idea about how cotton harvester works. Figure 7 shows CFPM.

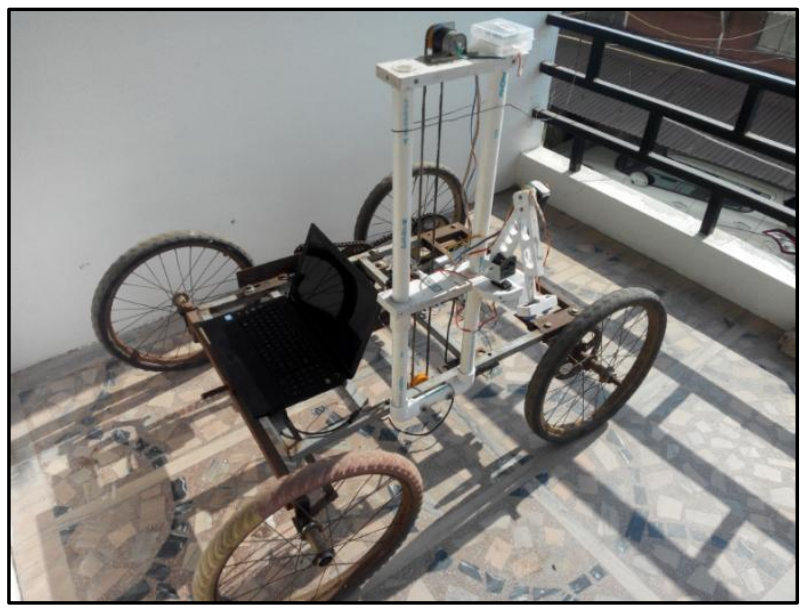

Figure 6 Cotton Flower Picking Machine

It include robotic arm to pick detected cotton. 4 wheel motion system is used for operation along farm. One vertical platform is used for mounting robotic arm and camera which move up and down for their operation.

\section{CONCLUSION}

In proposed work, development of cotton flower picking machine based on machine vision technique is presented. Results show that proposed method works good in various condition. All results are evaluated based on level of illumination on cotton plant. K- means clustering provide better accuracy in high sun as well as low sun light condition. Furthermore performance gain can be achieved by combining clustering algorithm with gamma correction or gamma adjusts operation. Machine vision system combined with motion control and robotic arm works well under supervision of computer as master controller. Robotic arm find some difficulty in appropriate positioning because of limited degree of freedom. This problem can be overcome by using robotic arm with more DOF which cover entire plant area. 


\section{REFERENCES}

[1] Wang, Mulan, Jieding Wei, Jianning Yuan, and Kaiyun $\mathrm{Xu}$. "A research for intelligent cotton picking robot based on machine vision." In Information and Automation, 2008. ICIA 2008. International Conference on, pp. 800-803. IEEE, 2008.

[2] Wang, Yong, Xiaorong Zhu, and Changying Ji. "Machine vision based cotton recognition for cotton harvesting robot." In International Conference on Computer and Computing Technologies in Agriculture, pp. 1421-1425. Springer, Boston, MA, 2007.

[3] Wang, Yong, Xiaorong Zhu, Yongxing Jia, and Changying Ji. "Object Recognition on Cotton Harvesting Robot Using Human Visual System." In International Conference on Computer and Computing Technologies in Agriculture, pp. 65-71. Springer, Berlin, Heidelberg, 2011

[4] Rao, U. S. N. "Design of automatic cotton picking robot with Machine vision using Image Processing algorithms." In Control, Automation, Robotics and Embedded Systems (CARE), 2013 International Conference on, pp. 1-5. IEEE, 2013.

[5] Bhattacharya, Mahua, Medhabi Verma, Vivek Shukla, S. S. Kohli, and P. Rajan. "Expert System Design for Cotton Harvesting Using Shape and Fractal Features." In Proceedings of the International Conference on Image Processing, Computer Vision, and Pattern Recognition (IPCV), p. 1. The Steering Committee of The World Congress in Computer Science, Computer
Engineering and Applied Computing (WorldComp), 2013.

[6] Li, Yanan, Zhiguo Cao, Hao Lu, Yang Xiao, Yanjun Zhu, and Armin B Cremers. "In-field cotton detection via region-based semantic image segmentation." Computers and Electronics in Agriculture 127 (2016): 475-486.

[7] Chin, Roland T., and Charles R. Dyer "Model-based recognition in robot vision." ACM Computing Surveys (CSUR)18, no. 1 (1986): 67-108.

[8] Sasaki, Hironobu, Naoyuki Kubota, Kousuke Sekiyama, and Toshio Fukuda. "Multiple object detection for intelligent robot vision by using growing neural gas." In Micro-NanoMechatronics and Human Science, 2009 MHS 2009. International Symposium on, pp. 80-85. IEEE, 2009.

[9] Wang, Yong, Ming-xia Shen, and Chang-ying Ji. "Study on the recognition of mature cotton based on the chromatic aberration in natural outdoor scenes." Acta Agriculturae Zhejiangensis 19, no. 5 (2007): 385

[10] Anjna, Er, and Er Rajandeep Kaur. "Review of Image Segmentation Technique." International Journal 8, no. 4 (2017).

[11] Wang, Weixin, Duanyang Qu, Benxue Ma, and Yage Wang. "Cotton Top feature identification based on machine vision \& image processing." In Computer Science and Automation Engineering (CSAE), 2011 IEEE International Conference on, vol. 1, pp. 681-685. IEEE, 2011 